\title{
The Utility of Endoscopic Ultrasound in Patients with Isolated Elevations in Serum Amylase and/or Lipase
}

\author{
Lalitha M. Sitaraman ${ }^{1}$, Amit H. Sachdev² ${ }^{2}$ Tamas A. Gonda ${ }^{2}$, Amrita Sethi ${ }^{2}$, John M. Poneros ${ }^{2}$ and Frank G. Gress ${ }^{2}$ \\ ${ }^{1}$ Division of Digestive Diseases, Department of Medicine, ${ }^{2}$ Division of Digestive and Liver Diseases, Columbia University Irving Medical \\ Center, New York, NY, USA
}

Background/Aims: The aim of this study was to describe the diagnostic yield of endoscopic ultrasound (EUS) in patients with isolated elevated levels of amylase and/or lipase.

Methods: A retrospective chart review was conducted at a large academic medical center from 2000 to 2016 . Patients were selected based on having elevated amylase, lipase, or both, but without a diagnosis of pancreatitis or known pancreatobiliary disease. Patients were excluded if they had abnormal liver function tests or abnormal imaging of the pancreas.

Results: Of 299 EUS procedures performed, 38 met inclusion criteria. Symptoms were present in 31 patients, most frequently abdominal pain (87\%). In 20 patients (53\%), initial EUS most commonly found chronic pancreatitis ( $n=7 ; 18 \%)$, sludge (5; 13\%), or new diagnosis of pancreas divisum (3; 8\%). In the asymptomatic patients (7), 3 had a finding on EUS, most importantly sludge (2), stone (1), and pancreas divisum (1). No patients were diagnosed with a mass or pancreatic cyst. During the follow up period, 6 patients (22\%) had cholecystectomy.

Conclusions: In our study of patients with isolated elevations in amylase and/or lipase without acute pancreatitis who underwent EUS, approximately $50 \%$ had a pancreatobiliary finding, most commonly chronic pancreatitis or biliary sludge. Clin Endosc 2019;52:175-181

Key Words: Endoscopic ultrasound; Endosonography; Pancreatitis; Amylase; Lipase

\section{INTRODUCTION}

Elevated amylase and lipase can represent pancreatic or non-pancreatic disease. ${ }^{1}$ Amylase is highly concentrated in the pancreas and the salivary glands, but other organs can make small amounts of amylase. Lipase is concentrated in the pancreas 5,000 times more than in other organs that can produce

Received: June 26, 2018 Revised: November 1, 2018

Accepted: November 2, 2018

Correspondence: Lalitha M. Sitaraman

Division of Digestive Diseases, Department of Medicine, Columbia University Irving Medical Center, 161 Fort Washington Avenue, Room 852A, New York, NY 10032, USA

Tel: +1-212-305-1909, Fax: +1-212-305-1081, E-mail: Lalitha.Sitaraman@gmail. com

ORCID: https://orcid.org/0000-0003-4867-1236

cc This is an Open Access article distributed under the terms of the Creative Commons Attribution Non-Commercial License (http://creativecommons.org/ licenses/by-nc/3.0) which permits unrestricted non-commercial use, distribution, and reproduction in any medium, provided the original work is properly cited. lipase. ${ }^{2}$ Elevations in amylase and lipase occur most commonly because of increased release from the pancreas into the bloodstream or decreased renal clearance of these enzymes. ${ }^{2}$ It is generally accepted that the mechanism of pancreatitis is premature activation of digestive enzymes within the acinar cell leading to autodigestion. ${ }^{3}$ In the evaluation of abdominal pain or other vague abdominal symptoms, pancreatic enzymes are frequently checked to exclude pancreatic disease.

However, elevations in amylase and/or lipase are not necessarily pathologic; macroamylasemia and chronic asymptomatic pancreatic hyperenzymemia $(\mathrm{CAPH})$ are benign conditions. The first description of chronic hyperamylasemia was published by Warshaw and Lee in 1978, where 17 patients had elevated amylase and no pancreatic disease. ${ }^{4}$ They concluded that macroamylasemia or extrapancreatic diseases may be responsible for this epiphenomenon. ${ }^{4}$ Years later, the syndrome was described as chronic nonpathological hyperamylasemia of pancreatic origin by Gullo in 1996, where 18 patients were 
assessed, and no pancreatic pathology was determined. ${ }^{5}$ However, Gullo's syndrome, also called benign pancreatic hyperenzymemia, is a diagnosis of exclusion and a clinician should be aware of various other conditions that can cause isolated elevation of pancreatic enzymes. ${ }^{6}$

In the absence of liver function abnormalities and noninvasive imaging abnormalities, evaluation and diagnosis of elevated pancreatic enzymes is enigmatic. The aim of this study was to better understand whether isolated mild elevations in amylase or lipase correlate with pancreatobiliary pathology and to determine the diagnostic yield of endoscopic ultrasound (EUS) in patients with otherwise normal imaging and isolated elevated amylase and/or lipase.

\section{MATERIALS AND METHODS}

After approval from the Columbia University Institutional Review Board, a query was performed to identify all cases of EUS from 2000 to 2016 with the indication of elevated amylase and/or lipase. This subset made up our study cohort, which was analyzed by retrospective chart review. Each subject record was reviewed, including consultation notes, progress notes, imaging reports, laboratory data, and endoscopy reports to determine if the patient met the criteria to be in the study group.

Patients were included if they had unexplained transient or persistent elevated amylase or lipase, less than 3 times normal values. Patients were excluded if they had known pancreatic disease (including acute or chronic pancreatitis [CP]), abnormal liver function tests (transaminases, bilirubin, or alkaline phosphatase), or prior abnormal imaging-ultrasound (US), computerized tomography (CT), or magnetic resonance cholangiopancreatography (MRCP). These exclusions occurred at any time in the chart review process. Patients with symptoms were not excluded.

The baseline characteristics collected included age, sex, any symptoms prior to EUS, and serum creatinine level. Additional data points collected prior to EUS were the serum levels of lipase and amylase, and if lipase and/or amylase was elevated on more than one occasion (i.e., persistently elevated) leading up to EUS. EUS was performed using radial scanning (EUM20; Olympus America, Inc., Center Valley, PA, USA) and a linear array (FG32UA; Pentax Instrument Co., Orangeburg, NY, USA) echoendoscope. Pathology reports resulting from the procedures were reviewed. The follow-up period was from the date of EUS going forward. Materials reviewed included IgG4 testing, imaging studies, any surgery, and endoscopic procedures-esophagogastroduodenoscopy (EGD), endoscopic retrograde cholangiopancreatography (ERCP), or EUS
- performed after initial EUS.

Abnormalities in the pancreas on EUS were assessed with the Rosemont Criteria, which requires major and minor criteria to classify CP. Major criteria for $\mathrm{CP}$ are hyperechoic foci with shadowing and main pancreatic duct calculi, and lobularity with honeycombing. Minor criteria for $\mathrm{CP}$ are the presence of cysts, dilated ducts $\geq 3.5 \mathrm{~mm}$, irregular duct contour, dilated side branches $\geq 1 \mathrm{~mm}$, hyperechoic duct walls, strands, nonshadowing hyperechoic foci, and lobularity with noncontiguous lobules. ${ }^{7}$ An example of CP on EUS is shown in Fig. 1. Endoscopists were not required to document all of the major and minor criteria that suggested $\mathrm{CP}$ in their reports.

Statistical analyses were calculated using chi-squared test with Stata software (StataCorp LLC, College Station, TX, USA).

\section{RESULTS}

Of 4,333 EUS procedures performed between 2000 and 2016, a total of 299 procedures were performed for the indication of elevated amylase or lipase; 38 patients met study inclusion criteria. The patients were predominantly female, and the majority were symptomatic at the time of EUS. The most common symptom was abdominal pain. Other symptoms and baseline characteristics are shown in Table 1.

Imaging studies were performed in the large majority (84\%) of patients (Fig. 2). Some patients (21\%) had a combination of studies (abdominal US, CT, MRCP), though MRCP was the only modality used in 18 patients (47\%). MRCP was not performed with secretin. No imaging studies had a clear diagnosis; those that were not completely normal were nonspecific or showed pancreas divisum.

Laboratory evaluation prior to EUS included collection of creatinine, liver function tests, and amylase and lipase (Table

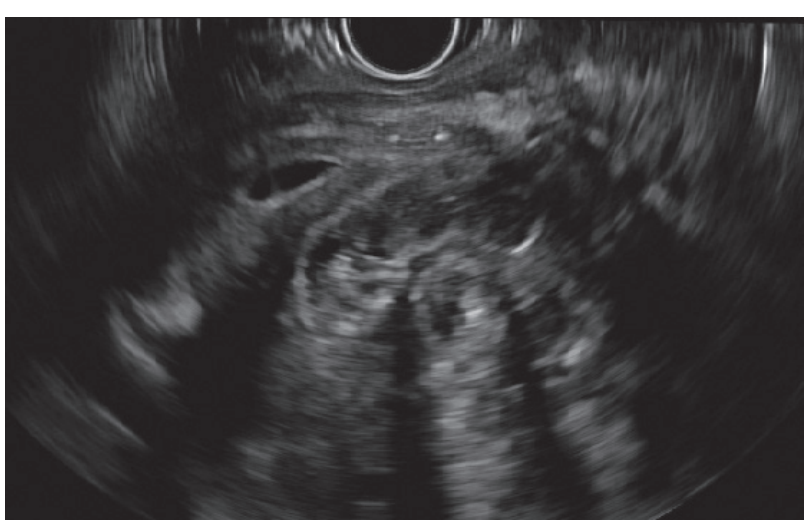

Fig. 1. Hyperechoic foci with shadowing-Major criteria for chronic pancreatitis on endoscopic ultrasound. 
1). Patients were excluded if they had abnormal liver function tests, so all liver function testing was within the normal range. Amylase and lipase values are summarized in Table 2. The majority of patients had elevation of lipase $(29,76 \%)$, with the majority having solitary elevation of lipase. There was documentation of persistent elevation, defined as more than 1 occasion, of lipase or amylase in 23 patients.

EUS was successfully completed in all 38 patients. There were no immediate complications, including no events of bleeding or perforation. EUS revealed at least 1 finding in 20 patients $(52.6 \%)$. The most common findings were $\mathrm{CP}$, sludge, pancreas divisum, and gallstones. Sludge was found in the gallbladder in 5 patients and the common bile duct in 2 patients. Stones were found in the cystic duct (1 patient) and the common bile duct (1 patient). Multiple diagnoses were found in some patients. All of the findings are summarized in Fig. 3. The Rosemont criteria were used to diagnose CP. Patients di-

Table 1. Baseline Characteristics

\begin{tabular}{lc}
\hline Age, yr (mean; range) & $50.3 ; 19-84$ \\
Sex & \\
$\quad$ Female & $29(74 \%)$ \\
$\quad$ Male & 9 \\
Symptoms & \\
$\quad$ Abdominal pain & $27(71 \%)$ \\
$\quad$ Diarrhea & 1 \\
Gas & 1 \\
$\quad$ Heartburn & 1 \\
$\quad$ Weight loss & 1 \\
$\quad$ No symptoms & $7(18 \%)$ \\
Creatinine, mg/dL (mean; range [SD]) & $0.896 ; 0.5-2.7(0.368)$ \\
\hline
\end{tabular}

$\mathrm{SD}$, standard deviation. agnosed with CP met major and minor criteria as required for diagnosis during EUS by the endoscopist, though documentation of the exact major and minor criteria was not required in reports. A new diagnosis of pancreas divisum was made in 3 patients.

Of the 7 patients with no symptoms, diagnoses were made in 3. Patient \#1 had mild atrophy but had no further follow-up. Patient \#2 had age-related changes in the pancreas and gallbladder sludge, and later required cholecystostomy for acute cholecystitis. Patient \#3 had a stone and sludge (in the common bile duct), and pancreas divisum, and required cholecystectomy. Patients \#1 and \#2 had isolated lipase elevation, and patient \#3 had elevations in both amylase and lipase.

Of the 27 patients with abdominal pain, diagnoses were made in 17 (44.7\%). The most common diagnosis was CP in 7 patients, followed by sludge (3) and pancreas divisum (3). During the follow-up period, cholecystectomy was performed in 2 of the 3 patients with sludge and 1 patient who had stones found on EUS.

An analysis of patients with elevated enzymes showed that those with increased lipase alone were found to have a diag-

Table 2. Amylase and Lipase Values

\begin{tabular}{lc}
\hline Lipase, U/L \\
Mean; Median (range) \\
Not elevated & $87.6 ; 72(49-246)$ \\
Amylase, U/L & 11 \\
$\quad$ Mean; Median (range) & \\
$\quad$ Not elevated & $165.9 ; 144(98-429)$ \\
Lipase alone elevated & 21 \\
Amylase alone elevated & 20 \\
Both elevated & 9 \\
\hline
\end{tabular}

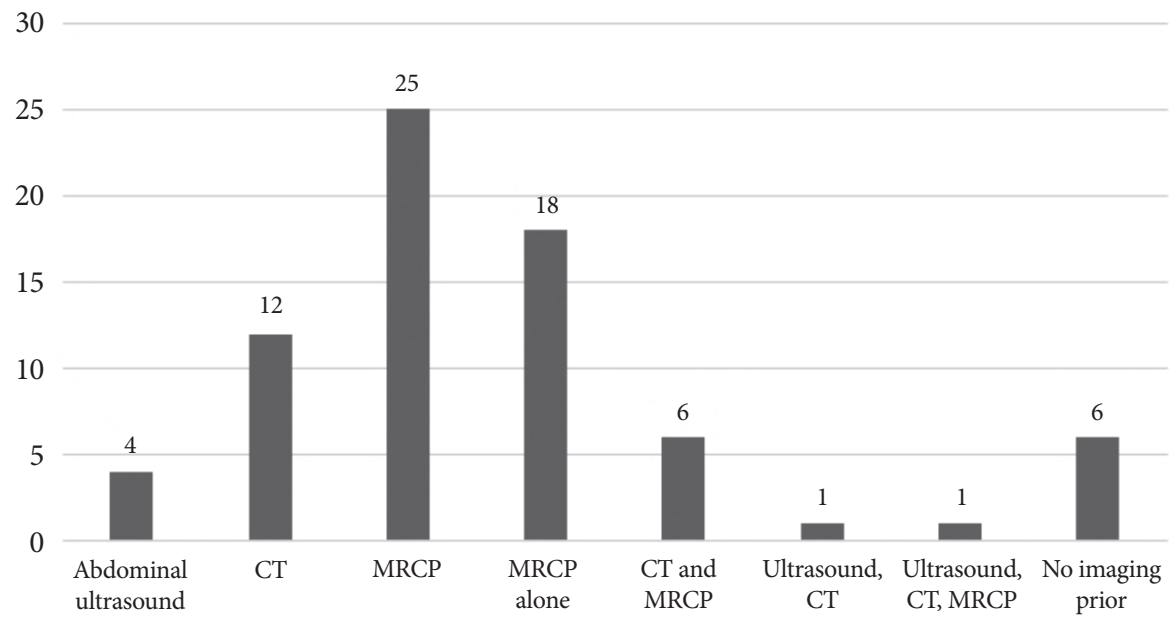

Fig. 2. Imaging modalities. CT, computerized tomography; MRCP, magnetic resonance cholangiopancreatography. 


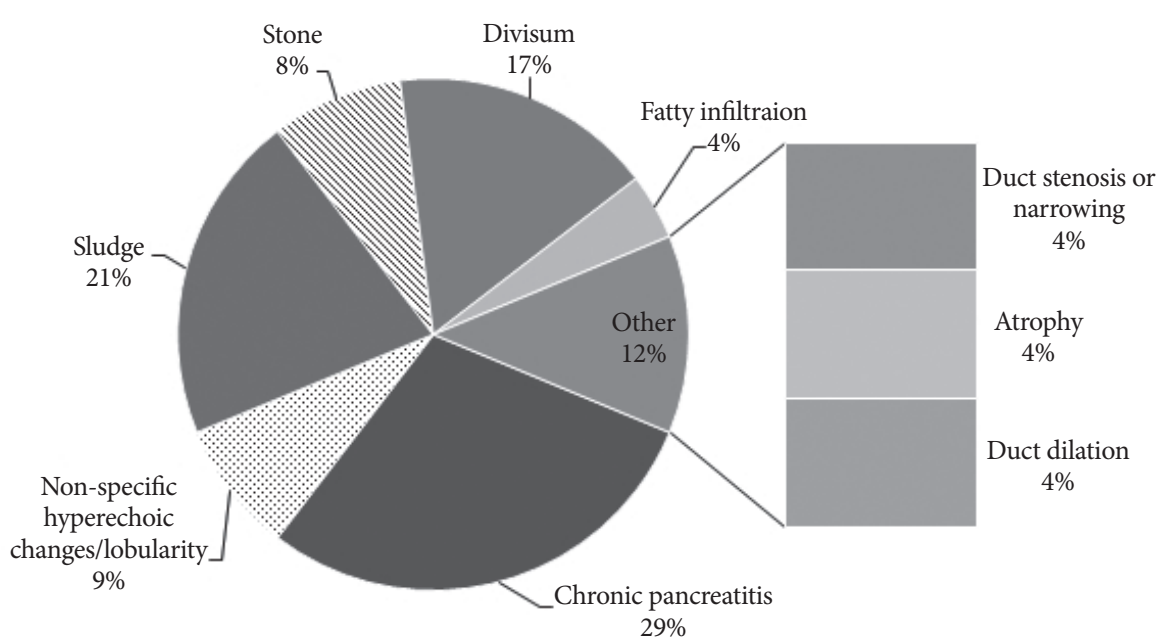

Fig. 3. Findings on endoscopic ultrasound.
Table 3. Findings Compared to Serum Test

\begin{tabular}{lcc}
\hline Serum elevation & Finding on EUS & No finding on EUS \\
\hline Amylase & 6 & 3 \\
Lipase & 9 & 11 \\
Both elevated & 5 & 4 \\
$p$-value & & 0.546 \\
\hline
\end{tabular}

EUS, endoscopic ultrasound.

nosis in 9 of $20(45 \%)$ cases, and in 6 of $9(67 \%)$ cases with isolated amylase elevation; if both were elevated, a diagnosis was found in 5 of $9(56 \%)$ cases. These differences were not statistically significant ( $p=0.546$ ) (Table 3 ). In the isolated elevated amylase group, 4 of 9 patients were screened for macroamylase (in urine or serum), and all were negative.

The majority of patients had some type of follow-up study, either repeat imaging or an endoscopic procedure. Eleven patients had no follow-up. The range of follow-up was 1 month to 10 years (median, 3 years). Imaging was performed in 20 patients, and repeat endoscopy procedures were performed in 18, either EGD (7), ERCP (6), or EUS (8). Over the course of the follow-up period, 1 patient developed acute pancreatitis and 1 developed acute cholecystitis. Five had cholecystectomy and 1 required cholecystostomy. One patient with sludge chose not to have cholecystectomy, and the other was lost to follow-up. Of the 6 patients with gallbladder intervention, 2 had normalization of lipase and amylase, 2 continued to have elevated enzymes, and 2 did not have amylase or lipase available postoperatively. Of the 6 patients who had ERCP, 4 were noted to have stenosis of the pancreatic duct. Of the 14 who were tested, IgG4 was elevated in 2. However, neither of these patients had signs of CP on EUS. One was normal and the other had findings consistent with age-related changes in the pancreas.
No patient in this cohort was found to have a pancreatic cyst, pseudocyst, intraductal papillary mucinous neoplasm (IPMN), or malignancy.

\section{DISCUSSION}

In general, it is believed that elevations in amylase and lipase denote pancreatic pathology. In the current study, patients with isolated mild pancreatic hyperenzymemia were studied. Patients with abnormalities in liver function tests, including bilirubin, were excluded to avoid confounding of liver disease, and patients with abnormal imaging were excluded because a diagnosis was already suggested. Given that all these patients were required to have normal imaging but not all were excluded on the basis of symptoms, this study has a unique cohort. Through literature review, it does not appear that a symptomatic group of patients with mild elevations in amylase or lipase has been studied before. Because our symptomatic patients did not have elevations in lipase greater than 3 times the upper limit of normal, those with abdominal pain do not fall under the definition of acute pancreatitis. However, the findings on EUS in our study suggest that about half the time these patients in this unique cohort did have pancreatobiliary pathology. The possible mechanisms will be discussed, along with the studies previously done in an asymptomatic cohort (patients with CAPH).

The most common findings on EUS in our cohort were $\mathrm{CP}$, biliary stones/sludge, and a new diagnosis of pancreas divisum.

The elevations of pancreatic enzymes in early $\mathrm{CP}$ are believed to be due to acinar cell damage, but these values can fluctuate and normal amylase or lipase does not exclude $\mathrm{CP}^{8}$ There are many theories about the pathogenesis of $\mathrm{CP}$, but in 
all of these cases, the main mechanism is acinar cell damage and resultant replacement with fibrotic tissues. ${ }^{9}$ Because the new tissue is nonfunctional, patients develop deficiency of the gland; both exocrine and endocrine functions can be affected. $^{10}$

Many of our patients had signs of CP on EUS. The Rosemont classification was used for diagnosis, which includes meeting major and minor criteria on EUS. ${ }^{7}$ However, the diagnosis of $\mathrm{CP}$ is complex in general due to varying definitions. ${ }^{11}$ The American Pancreatic Association published guidelines for diagnosis in 2011 and 2014 that involve a stepwise process for diagnosis, which includes clinical signs and symptoms as well as noninvasive and invasive imaging. ${ }^{12}$ The incidence of $\mathrm{CP}$ has been studied in the United States; a population-based study showed an incidence of 4.35 per 100,000 person-years from $1997-2006 .{ }^{13}$ In that study, some cases were diagnosed only at autopsy.

In the current cohort of patients, all of those diagnosed with $\mathrm{CP}$ had abdominal pain, but signs of exocrine deficiency, such as steatorrhea, were not documented in data available at the time of this study. In the absence of pancreatic dysfunction, it is difficult to diagnose these patients with true CP. The high incidence of CP in our cohort, 7 patients, is likely due to multiple reasons. The fact that ours is a tertiary care referral center and that many of these patients were referred for EUS after unrevealing prior workup selects a specific group of patients. Additionally, given our findings, the incidence rate of $\mathrm{CP}$, especially early or mild $\mathrm{CP}$, may actually be higher than has been reported. Our patients may have had early enough $\mathrm{CP}$ to have elevated enzymes as a sign of cellular damage but not severe enough to have exocrine deficiency.

In patients with acute pancreatitis due to gallstones, the mechanism of elevated serum amylase and lipase is believed to be the transient obstruction of the pancreatic duct, causing an increase in pressure within the duct and backup of pancreatic enzymes. ${ }^{3}$ In our patient cohort, 7 patients had gallstones or sludge. Based on this theory, the mechanism in our patients could also be the transient obstruction of the pancreatic duct by stones or sludge. The stones and sludge were located in either or both the common bile duct and gallbladder. In the follow-up period, 6 patients had cholecystectomy or cholecystostomy, either prophylactically for acute cholecystitis, or for gallstone pancreatitis. Five of these patients had stones or sludge detected on initial EUS. Of the 2 of the initial 7 who did not have cholecystectomy in our cohort, 1 patient with sludge was lost to follow up, and the other was referred for cholecystectomy but chose not to have surgery. Amylase and lipase were available in 4 of the patients after surgery, and 2 had complete resolution of elevated enzymes. The improvement in enzymes also supports that stones and sludge could be the reason for elevated enzymes in our cohort.

The association with pancreas divisum and pancreatitis is controversial. Since an early description in 1980, multiple theories and evidence supporting and refuting have been presented. ${ }^{14-17}$ The theory presented most commonly is that patients with pancreas divisum develop obstruction of the accessory papilla independently, which causes acute pancreatitis. Multiple studies have found that patients with recurrent pancreatitis have a higher rate of pancreas divisum, and pancreas divisum is associated with $\mathrm{CP}^{15}$ The finding of pancreas divisum in our cohort, newly diagnosed in 3 patients, could also potentially be explained by intermittent obstruction, which would support the theory that pancreas divisum is a risk factor for acute and CP.

Asymptomatic patients with elevated pancreatic enzymes have been studied. The first major study of 18 patients was published by Gullo in $1996 .{ }^{5}$ In that study, CT and abdominal US were used to exclude other etiologies of hyperenzymemia. ${ }^{5}$ After CAPH was first described, a number of studies have tried to explain the condition as more than idiopathic. In a recent study, patients with CAPH were imaged with secretin-MRCP (s-MRCP) or EUS and diagnoses were found in $63.3 \%$ of 68 patients when both tests were combined. ${ }^{18}$ In that study, EUS alone found a diagnosis in $60.3 \%$ of patients, and independently found a diagnosis in $9 \%$. In the present study, the yield of EUS found a unique diagnosis in 14 of the $25 \mathrm{pa}-$ tients who had normal MRCP prior to EUS, which at $56 \%$ is much higher. Thus, CAPH is not always a benign syndrome with no pancreatobiliary manifestations, and EUS can be a diagnostic tool in patients with prior unrevealing noninvasive workup.

In our cohort of asymptomatic patients, EUS discovered 3 findings in 7 patients (42.8\%): sludge (2), stone (1), and pancreas divisum (1). Two of those 3 patients had surgical intervention on the gallbladder in the follow-up period (cholecystectomy and cholecystostomy); 1 was for acute cholecystitis and the other was preventative. In another study with CAPH patients, s-MRCP showed abnormal imaging findings in 50\% and clinically significant results in $14.4 \%$; those patients had surgery for pancreatic tumor or follow-up for IPMN..$^{19}$ None of our patients had masses or IPMN, likely because their MRCP was normal. Both of the asymptomatic patients who had biliary surgery had MRCP prior to EUS. Of 4 symptomatic patients who had cholecystectomy, 2 had MRCP before surgery, 1 of whom developed gallstone pancreatitis. The missed diagnoses on MRCP resulted in clinically significant changes in management.

The patients with amylase elevation alone had a higher rate of diagnosis in our study, but this was not statistically significant (Table 3). Macroamylasemia can sometimes 
explain persistently elevated amylase, and it can be seen in other conditions, such as celiac disease, inflammatory bowel disease, and autoimmune diseases like systemic lupus erythematosus. ${ }^{2,20}$ Although testing for macroamylasemia was not specifically performed in every patient in this cohort, all 4 of the 9 patients with elevated amylase alone who were tested did not have macroamylase present in urine or serum. Given that those with elevated amylase had a higher percentage of EUS findings as well, this suggests that macroamylase was not a contributing factor for persistent hyperamylasemia in our cohort.

Studies have also shown that chronic kidney disease can elevate amylase and lipase levels. ${ }^{2}$ However, in our study, only 4 of 38 patients had elevated creatinine (when defined as greater than $1.2 \mathrm{mg} / \mathrm{dL}$, the upper limit of normal at our laboratory). Of those patients with renal insufficiency, 3 of the 4 had a finding on EUS. The 1 patient without any EUS finding had both amylase and lipase elevation with a creatinine of $2.7 \mathrm{mg} /$ dL. We did not exclude patients with renal insufficiency because not all have elevated pancreatic enzymes. ${ }^{21}$

The current study has some limitations. Specifically, this study is limited by not having a medication history, ${ }^{22,23}$ human immunodeficiency virus history, ${ }^{24}$ or alcohol use history, ${ }^{25}$ which can all contribute to elevated pancreatic enzyme levels. Additionally, our patients were not screened for SPINK1 and PRSS1 mutations, which may have an effect on amylase and lipase levels, though Gullo did not show any significance for these mutations. ${ }^{6}$ Our patients did not have s-MRCP. Because the study was retrospective, the workup before EUS was not standardized, so not every patient had the same imaging modality prior to EUS. Our study was also limited by the fact that there is no gold standard for imaging the pancreas or confirming diagnoses seen on EUS. Finally, given that some of these patients were referred to our large academic hospital, the extent of additional testing done prior to or after EUS was also limited, which also partially accounts for the lack of follow-up available on some of the patients in this retrospective study.

Though EUS is an invasive procedure, it has a low complication rate as a diagnostic tool. The safety of EUS alone as a diagnostic procedure is not as well studied as EUS in conjunction with interventional procedures, such as fine-needle aspiration. ${ }^{26}$ However, a study involving the review of 43,852 patients found only $16(0.03 \%)$ had perforation in the cervical esophagus. ${ }^{27}$ None of the patients in our study had bleeding, perforation, infection, or other complications of EUS. However, patients should be carefully selected for EUS, and both the risks and benefits should be discussed with patients prior to the procedure.

In conclusion, EUS does have diagnostic yield in patients with isolated elevated amylase and/or lipase, and provided a probable diagnosis in $53 \%$ of those with previously normal imaging. Asymptomatic patients also can have findings that are not seen on noninvasive imaging modalities. Correctly ruling out pancreaticobiliary pathology is important to minimize healthcare costs and allay patient anxiety. EUS may also play a role earlier in the diagnostic process given the high yield of diagnoses found in this study, especially in CP. Future studies are now needed to investigate these findings in a more controlled study design. Ideally, these future studies should also follow patients prospectively to determine the long-term outcomes of patients with persistent mildly-elevated pancreatic enzymes.

\section{Conflicts of Interest}

The authors have no financial conflicts of interest.

\section{REFERENCES}

1. Muniraj T, Dang S, Pitchumoni CS. Pancreatitis or not?--elevated lipase and amylase in ICU patients. J Crit Care 2015;30:1370-1375.

2. Frulloni L, Patrizi F, Bernardoni L, Cavallini G. Pancreatic hyperenzymemia: clinical significance and diagnostic approach. JOP 2005;6:536551.

3. Vonlaufen A, Wilson JS, Apte MV. Molecular mechanisms of pancreatitis: current opinion. J Gastroenterol Hepatol 2008;23:1339-1348.

4. Warshaw AL, Lee KH. Macroamylasemia and other chronic nonspecific hyperamylasemias: chemical oddities or clinical entities? Am J Surg 1978;135:488-493

5. Gullo L. Chronic nonpathological hyperamylasemia of pancreatic origin. Gastroenterology 1996;110:1905-1908.

6. Gullo L. Benign pancreatic hyperenzymemia. Dig Liver Dis 2007;39:698702 .

7. Catalano MF, Sahai A, Levy M, et al. EUS-based criteria for the diagnosis of chronic pancreatitis: the Rosemont classification. Gastrointest Endosc 2009;69:1251-1261.

8. Ito T. Can measurement of chemokines become useful biological and functional markers of early-stage chronic pancreatitis? J Gastroenterol 2007;42 Suppl 17:72-77.

9. Stevens T, Conwell DL, Zuccaro G. Pathogenesis of chronic pancreatitis: an evidence-based review of past theories and recent developments. Am J Gastroenterol 2004;99:2256-2270.

10. Duggan SN. Negotiating the complexities of exocrine and endocrine dysfunction in chronic pancreatitis. Proc Nutr Soc 2017;76:484-494.

11. Duggan SN, Ni Chonchubhair HM, Lawal O, O'Connor DB, Conlon KC. Chronic pancreatitis: a diagnostic dilemma. World J Gastroenterol 2016;22:2304-2313.

12. Conwell DL, Lee LS, Yadav D, et al. American pancreatic association practice guidelines in chronic pancreatitis: evidence-based report on diagnostic guidelines. Pancreas 2014;43:1143-1162.

13. Yadav D, Timmons L, Benson JT, Dierkhising RA, Chari ST. Incidence, prevalence, and survival of chronic pancreatitis: a population-based study. Am J Gastroenterol 2011;106:2192-2199.

14. Cotton PB. Congenital anomaly of pancreas divisum as cause of obstructive pain and pancreatitis. Gut 1980;21:105-114.

15. DiMagno MJ, Wamsteker EJ. Pancreas divisum. Curr Gastroenterol Rep 2011:13:150-156.

16. Adike A, El Kurdi BI, Gaddam S, et al. Pancreatitis in patients with pan- 
creas divisum. Pancreas 2017;46:e80-e81.

17. Burtin P, Person B, Charneau J, Boyer J. Pancreas divisum and pancreatitis: a coincidental association? Endoscopy 1991;23:55-58.

18. Di Leo M, Petrone MC, Zuppardo RA, et al. Pancreatic morpho-functional imaging as a diagnostic approach for chronic asymptomatic pancreatic hyperenzymemia. Dig Liver Dis 2016;48:1330-1335.

19. Amodio A, Manfredi R, Katsotourchi AM, et al. Prospective evaluation of subjects with chronic asymptomatic pancreatic hyperenzymemia. Am J Gastroenterol 2012;107:1089-1095.

20. Berk JE, Kizu H, Wilding P, Searcy RL. Macroamylasemia: a newly recognized cause for elevated serum amylase activity. N Engl J Med 1967;277:941-946.

21. Völzke H, Lüdemann J, Mayerle J, Kraft M, John U, Lerch MM. Prevalence and determinants of increased serum lipase levels in a general population. Pancreas 2008;37:411-417.

22. Sharma A, Masood U, Khan B, Chawla K, Manocha D. Pancreatitis with normal lipase and amylase in setting of end-stage renal disease. Am J Emerg Med 2017;35:1387.e3-1387.e4.

23. Ustohal L, Mayerova M, Valkova B, Sedlakova H, Kasparek T. Asymptomatic elevation of amylase and lipase after olanzapine treatment. J Clin Psychopharmacol 2016;36:181-183.

24. Szoke D, Ridolfo A, Valente C, Galli M, Panteghini M. Frequency of pancreatic hyperamylasemia in human immunodeficiency virus-positive patients in the highly active antiretroviral therapy era. Am J Clin Pathol 2016;145:128-133.

25. Pezzilli R. Alcohol abuse and pancreatic diseases: an overview. Recent Pat Inflamm Allergy Drug Discov 2015;9:102-106.

26. Saumoy M, Kahaleh M. Safety and complications of interventional endoscopic ultrasound. Clin Endosc 2018;51:235-238.

27. Das A, Sivak MV Jr, Chak A. Cervical esophageal perforation during EUS: a national survey. Gastrointest Endosc 2001;53:599-602. 